\title{
Analysis of Progressive First-Failure-Censoring for Non-normal Model Using Competing Risks Data
}

\author{
A. A. Modhesh ${ }^{1}$, G. A. Abd-EImougod ${ }^{2}$ \\ ${ }^{1}$ Department of Mathematics, Faculty of Science, Taiz University, Taiz, Yemen \\ ${ }^{2}$ Department of Mathematics, Faculty of Science, Taif University, KSA
}

Email address:

a_a_mod@yahoo.com (A. A. Modhesh)

To cite this article:

A. A. Modhesh, G. A. Abd-Elmougod. Analysis of Progressive First-Failure-Censoring for Non-normal Model Using Competing Risks Data. American Journal of Theoretical and Applied Statistics. Vol. 4, No. 6, 2015, pp. 610-618. doi: 10.11648/j.ajtas.20150406.33

\begin{abstract}
Competing risks data usually arises in studies in which the death or failure of an individual or an item may be classified into one of $\mathrm{T} \geq 2$ mutually exclusive causes. In this paper, we will study the competing risks model when the data is progressively first-failure-censored. Based on this type of censoring, we derive the maximum likelihood estimators (MLE's) for the unknown parameters. Approximate confidence intervals and two bootstrap confidence intervals are also proposed. The results in the cases of first-failure censoring, progressive Type II censoring, Type II censoring and complete sample are special cases. A real data set has been analyzed for illustrative purposes. Different methods have been compared using Monte Carlo simulations.
\end{abstract}

Keywords: Burr XII Distribution, Progressive First-Failure-Censoring, Competing Risks, Maximum Likelihood Method, Bootstrap

\section{Introduction}

In medical studies and reliability analyses an investigator is often interested in the assessment of a specific risk in the presence of other risk factors. In statistical literature this is known as the analysis of competing risks model. A lifetime experiment with $\tau=2$ different risk factors competing for the failure of the experimental units is considered. The data for such a competing risks model consist of the lifetime of the failed item and an indicator variable which denotes the cause of failure. For example, the competing risk for a prostate cancer patient may include prostate cancer itself, heart disease and (all) other causes. The effects of the other competing risks may play an important role in survival studies on slowly progressing diseases such as prostate cancer. In engineering applications, the causes or risks may signify either multiple modes of failure for a complex unit or multiple components or subsystems which comprise an entire system. Occurrence of a system failure is caused by the earliest onset of any of these component failures. In this respect, the framework is that of a system with components connected in series. Several studies have been carried out under this assumption and the risks follow different lifetime distributions, namely the exponential, lognormal, gamma, Weibull, generalized exponential or exponentiated Weibull; see for example Moeschberger et al.
[1], Pascual [2] Cramer and Schmiedt [3], Sarhan et al. [4], Sarhan [5], Alwasel [6], Kundu and Bas [7] and Kundu and Sarhan [8].

Censoring occurs when exact lifetimes are known only for a portion of the individuals or units under study, while for the remainder of the lifetimes information on them is partial. There are several types of censored tests. The most common censoring schemes are Type-I (time) censoring, where the life testing experiment will be terminated at a prescribed time $T$, and Type-II (failure) censoring, where the life testing experiment will be terminated upon the $r$-th ( $r$ is pre-fixed) failure. However, the conventional Type-I and Type-II censoring schemes do not have the flexibility of allowing removal of units at points other than the terminal point of the experiment. A generalization of Type II censoring is the progressive Type II censoring. It is a method which enables an efficient exploitation of the available resources by continual removal of a prespecified number of surviving test units at each failure time. On other hand, the removal of units before failure may be intentional to save time and cost or when some items have to be removed for use in another experiment. Wu et al. [9] and $\mathrm{Wu}$ and $\mathrm{Yu}$ [10] for extensive reviews of the literature on progressive censoring. When the lifetimes of products are very high, the experimental time of a Type II censoring life test can be still too long. Because of these lack of flexibilities, Johnson 
[11] described a life test in which the experimenter might decide to group the test units into several sets, each as an assembly of test units, and then run all the test units simultaneously until occurrence the first failure in each group. Such a censoring scheme is called first-failure censoring. If an experimenter desires to remove some sets of test units before observing the first-failures in these sets this life test plan is called progressive first-failure-censoring scheme.

In this section, first-failure censoring is combined with progressive censoring scheme as in Wu and Kuş [12] Suppose that $n$ independent groups with $k$ items within each group are put on a life test, $R_{1}$ groups and the group in which the first failure is observed are randomly removed from the test as soon as the first failure (say $X_{1: m: n: k}^{\mathbf{R}}$ and $\delta_{1} \in\{1,2\}$ ) has occurred, $R_{2}$ groups and the group in which the second first failure is observed are randomly removed from the test when the second failure (say $X_{2: m: n: k}^{\mathbf{R}}$ and $\delta_{2} \in\{1,2\}$ ) has occurred, and finally $R_{m}(m \leq n)$ groups and the group in which the $m$-th first failure is observed are randomly

removed from the test as soon as the $m$-th failure (say $X_{m: m: n: k}^{\mathbf{R}}$ and $\left.\delta_{m} \in\{1,2\}\right)$ has occurred. The data $\left(X_{1: m: n: k}^{\mathbf{R}}, \delta_{1}\right)<\left(X_{2: m: n: k}^{\mathbf{R}}, \delta_{2}\right)<\ldots<$ $\left(X_{m: m: n: k}^{\mathbf{R}}, \delta_{m}\right) \quad$ are called progressively first-failure-censored order statistics with the progressive censoring scheme $\mathbf{R}=\left(R_{1}, \ldots, R_{m}\right)$ and for each $i, \delta_{i}$ takes a value either 1 and 2 the causes of failures. It is clear that $n=m+R_{1}+R_{2}+\ldots+R_{m}$. For a given censoring scheme $\mathbf{R}=\left\{R_{1}, \ldots, R_{m}\right\}$, the likelihood function of the observed data $\left(X_{1: m: n: k}^{\mathbf{R}}, \delta_{1}\right)<\left(X_{2: m: n: k}^{\mathbf{R}}, \delta_{2}\right)<\ldots<$ $\left(X_{m: m: n: k}^{\mathbf{R}}, \delta_{m}\right)$

$$
\begin{aligned}
\ell\left(\alpha, \beta_{1}, \beta_{2} ; \underline{x}\right)= & C \prod_{i=1}^{m}\left[H_{1}\left(x_{i ; m, n, k}^{\mathbf{R}}\right)\right]^{I\left(\delta_{i}=1\right)}\left[H_{2}\left(x_{i ; m, n, k}^{\mathbf{R}}\right)\right]^{I\left(\delta_{i}=2\right)} \\
& \times\left[S_{1}\left(x_{i ; m, n, k}^{\mathbf{R}}\right) S_{2}\left(x_{i ; m, n, k}^{\mathbf{R}}\right)\right]^{k\left(R_{i}+1\right)}
\end{aligned}
$$

$$
0<\left(X_{1: m: n: k}^{\mathbf{R}}, \delta_{1}\right)<\left(X_{2: m: n: k}^{\mathbf{R}}, \delta_{2}\right)<\ldots<\left(X_{m: m: n: k}^{\mathbf{R}}, \delta_{m}\right)<\infty
$$

where $S(t)$ and $H(t)$ are reliability and failure rate functions, respectively, and

$$
C=n\left(n-R_{1}-1\right)\left(n-R_{1}-R_{2}-2\right) \ldots\left(n-R_{1}-R_{2}-\ldots-R_{m-1}-m+1\right) k^{m} .
$$

\section{Special cases}

It is clear from (1) that the progressive first-failure-censored scheme containing the following censoring schemes as special cases:

The first-failure censored scheme when $\mathbf{R}=\{0,0, \ldots, 0\}$.

The progressive Type II censored order statistics if $k=1$.

Usually Type II censored order statistics when $k=1$ and $\mathbf{R}=\{0,0, \ldots, n-m\}$.

The complete sample case when $k=1$ and $\mathbf{R}=\{0,0, \ldots, 0\}$.

For more applications about progressive-first-failure censoring data the readers may refer to Soliman et al [13] Soliman et al. ([14], [15]), Soliman et al. [16] and Ahmadi et al. [17].

The main aim of this paper is to develop a confidence interval and the MLE for the Burr XII parameters based on the progressively first-failure-censored sample in the presence of competing risks. Therefore, the organization of the paper is as follows. The model description and notation used throughout this paper are introduced in Section 2. The MLE's of the unknown parameters are presented in Section 3. Approximate confidence intervals and two parametric bootstrap confidence intervals are discussed in Section 4. A real data set, due to Hoel [18], is analyzed in Section 5. In Section 6, the different methods are compared by conducting Monte Carlo simulations.
Some concluding remarks are finally made in Section 7.

\section{Model Assumptions and Notations}

Before proceeding any further, we describe some notations we are going to use in this paper.

lifetime of the $i$-th unit.

lifetime of the $i$-th individual under cause $j, j=1,2$.

cumulative distribution function (cdf) of $X_{i}$.

probability density function (pdf) of $F($.$) .$

distribution function (cdf) of $X_{i j}$.

probability density function (pdf) of $F_{j}($.$) .$

survival function of $X_{i j}$.

indicator variable denoting the cause of failure of the $i$-th individual.

To simplify the notations we will use hereafter $X_{i}$ instead of $X_{i: m: n: k}^{\mathbf{R}}, i=1,2, \ldots, m$. The model studied in the paper satisfies the following assumptions

I) The lifetime of unit is denoted as $X_{i}, i=1,2, \ldots, m$. The time at which the unit $i$ fails due to cause $j$ is $X_{i j}$, $j=1,2$. That is, $X_{i}=\min \left\{X_{i 1}, X_{i 2}\right\}$. 
II) The distribution of the random variable $X_{i j}$ is Burr XII with shape parameters $\alpha$ and $\beta_{j}, j=1,2$ and $i=1,2, \ldots, m$. That is, the (pdf) and (cdf) of $X_{i j}, j=1,2$, and $i=1,2, \ldots, m$, are

$$
\begin{gathered}
f_{j}(x)=\alpha \beta_{j} x^{\alpha-1}\left(1+x^{\alpha}\right)^{-\left(\beta_{j}+1\right)}, x>0,\left(\alpha>0, \beta_{j}>0\right), \\
F_{j}(x)=1-\left(1+x^{\alpha}\right)^{-\beta_{j}}, x>0 .
\end{gathered}
$$

The corresponding reliability and failure rate functions of the Burr XII distribution at some $t$, are

$$
\begin{gathered}
S_{j}(t)=\left(1+t^{\alpha}\right)^{-\beta_{j}}, t>0, \\
H_{j}(t)=\alpha \beta_{j} t^{\alpha-1}\left(1+t^{\alpha}\right)^{-1}, t>0,
\end{gathered}
$$

respectively.

The two-parameter Burr XII distribution has unimodal or decreasing failure rate function Eq. (6). It is clear that the parameter $\beta_{j}$ does not affect the shape of failure rate function $H_{j}(t)$ and $\alpha$ is the shape parameter. Thus the shape parameter $\alpha$ plays an important role. Its capacity to assume various shapes often permits a good fit for describing biological, clinical or other experimental data sets.

\section{Maximum Likelihood Estimation}

Based on the observed sample $\left(x_{1: m: n}, \delta_{1}, R_{1}\right)$, $\left(x_{2: m: n}, \delta_{2}, R_{2}\right), \cdots,\left(x_{m: m: n}, \delta_{m}, R_{m}\right)$, the likelihood function (1), is given by

$$
\ell\left(\alpha, \beta_{1}, \beta_{2} ; \underline{x}\right) \propto \alpha^{m} \beta_{1}^{m_{1}} \beta_{2}^{m_{2}} \eta(\alpha ; \underline{x}) \exp \left[-k\left(\beta_{1}+\beta_{2}\right) \sum_{i=1}^{m}\left(R_{i}+1\right) \log \left(1+x_{i}^{\alpha}\right)\right]
$$

where

$$
\eta(\alpha ; \underline{x})=\prod_{i=1}^{m} \frac{x_{i}^{\alpha-1}}{\left(1+x_{i}^{\alpha}\right)} .
$$

The log-likelihood function without the additive constant can be written as follows;

$$
\begin{aligned}
L\left(\alpha, \beta_{1}, \beta_{2} ; \underline{x}\right)= & m \log \alpha+m_{1} \log \beta_{1}+m_{2} \log \beta_{2}+(\alpha-1) \sum_{i=1}^{m} \log \left(x_{i}\right) \\
& -\sum_{i=1}^{m} \log \left(1+x_{i}^{\alpha}\right)-k\left(\beta_{1}+\beta_{2}\right) \sum_{i=1}^{m}\left(R_{i}+1\right) \log \left(1+x_{i}^{\alpha}\right) .
\end{aligned}
$$

Calculating the first partial derivatives of (9) with respect to $\beta_{1}$ and $\beta_{2}$ and equating to zero, we obtain the likelihood equations

$$
\hat{\beta}_{2}(\alpha)=\frac{m_{2}}{k \sum_{i=1}^{m}\left(R_{i}+1\right) \log \left(1+x_{i}^{\alpha}\right)} .
$$

$$
\frac{\partial L\left(\underline{x} ; \alpha, \beta_{1}, \beta_{2}\right)}{\partial \beta_{1}}=\frac{m_{1}}{\beta_{1}}-k \sum_{i=1}^{m}\left(R_{i}+1\right) \log \left(1+x_{i}^{\alpha}\right)=0,
$$

and

$$
\frac{\partial L\left(\underline{x} ; \alpha, \beta_{1}, \beta_{2}\right)}{\partial \beta_{2}}=\frac{m_{2}}{\beta_{2}}-k \sum_{i=1}^{m}\left(R_{i}+1\right) \log \left(1+x_{i}^{\alpha}\right)=0 .
$$

Hence, the MLE's of $\beta_{1}$ and $\beta_{2}$, respectively, given by

$$
\hat{\beta}_{1}(\alpha)=\frac{m_{1}}{k \sum_{i=1}^{m}\left(R_{i}+1\right) \log \left(1+x_{i}^{\alpha}\right)},
$$

and

Using (12) and (13) in (9), we obtain the profile likelihood function for $\alpha$, as

$$
\begin{aligned}
& p(\alpha)=m \log \alpha-m \log \left[k \sum_{i=1}^{m}\left(R_{i}+1\right) \log \left(1+x_{i}^{\alpha}\right)\right] \\
& +\alpha \sum_{i=1}^{m} \log \left(x_{i}\right)-\sum_{i=1}^{m} \log \left(1+x_{i}^{\alpha}\right) .
\end{aligned}
$$

Therefore, the MLE of $\alpha$, say $\hat{\alpha}$, can be obtained by maximizing (14) with respect to $\alpha$. The MLE $\hat{\alpha}$ which maximizes (14) can be obtained from

$$
h(\alpha)=\alpha,
$$

where 


$$
h(\alpha)=m\left[\frac{m \sum_{i=1}^{m} \frac{\left.\left(R_{i}+1\right)\right)_{i}^{a_{i} \log \left(x_{i}\right)}}{\left(1+x_{i}^{\alpha}\right)}}{\sum_{i=1}^{m}\left(R_{i}+1\right) \log \left(1+x_{i}^{\alpha}\right)}+\sum_{i=1}^{m} \frac{x_{i}^{\alpha} \log \left(x_{i}\right)}{\left(1+x_{i}^{\alpha}\right)}-\sum_{i=1}^{m} \log \left(x_{i}\right)\right]^{-1}
$$

Thus, the MLE $\hat{\alpha}$ of the parameter $\alpha$ can be obtained by solving the nonlinear Eq. (15) using, for example, the Newton-Raphson or fixed point iteration. The corresponding MLE $\hat{\beta}_{1}$ and $\hat{\beta}_{2}$ of the parameters $\beta_{1}$ and $\beta_{2}$ are computed from Equations (12) and (13).

Notice that both $m_{1}$ and $m_{2}$ follow binomial distributions with sample size $m$. Hence, $m_{i} \sim$ Bin $\left(m, \beta_{i} /\left(\beta_{1}+\beta_{2}\right)\right), \quad i=1,2$.

\section{Confidence Intervals}

In this section, we propose different confidence intervals. One is based on the asymptotic distribution of $\hat{\alpha}, \hat{\beta}_{1}$ and $\hat{\beta}_{2}$ and two bootstrap confidence intervals.

\subsection{Approximate Confidence Intervals}

From the log-likelihood function in (9), we have

$$
\begin{gathered}
\frac{\partial^{2} L}{\partial \alpha^{2}}=-\frac{m}{\alpha^{2}}-\sum_{i=1}^{m} \frac{x_{i}^{\alpha} \log ^{2}\left(x_{i}\right)}{\left(1+x_{i}^{\alpha}\right)^{2}}-k\left(\beta_{1}+\beta_{2}\right) \sum_{i=1}^{m} \frac{\left(R_{i}+1\right) x_{i}^{\alpha} \log ^{2}\left(x_{i}\right)}{\left(1+x_{i}^{\alpha}\right)^{2}}, \\
\frac{\partial^{2} L}{\partial \alpha \partial \beta_{1}}=\frac{\partial^{2} L}{\partial \beta_{1} \partial \alpha}=-k \sum_{i=1}^{m} \frac{\left(R_{i}+1\right) x_{i}^{\alpha} \log \left(x_{i}\right)}{\left(1+x_{i}^{\alpha}\right)} \\
\frac{\partial^{2} L}{\partial \alpha \partial \beta_{2}}=\frac{\partial^{2} L}{\partial \beta_{2} \partial \alpha}=-k \sum_{i=1}^{m} \frac{\left(R_{i}+1\right) x_{i}^{\alpha} \log \left(x_{i}\right)}{\left(1+x_{i}^{\alpha}\right)} \\
\frac{\partial^{2} L}{\partial \beta_{1} \partial \beta_{2}}=\frac{\partial^{2} L}{\partial \beta_{2} \partial \beta_{1}}=0
\end{gathered}
$$

and

$$
\frac{\partial^{2} L}{\partial \beta_{1}^{2}}=-\frac{m_{1}}{\beta_{1}^{2}}, \quad \frac{\partial^{2} L}{\partial \beta_{2}^{2}}=-\frac{m_{1}}{\beta_{2}^{2}} .
$$

To find the confidence interval for the estimators we determine the asymptotic distribution of the maximum likelihood estimator of the element of the vector of unknown parameters $\theta=\left(\alpha, \beta_{1}, \beta_{2}\right)$, which produce an approximation confidence interval. It is known that the asymptotic distribution of the MLE is given by

$$
\left((\hat{\alpha}-\alpha),\left(\hat{\beta}_{1}-\beta_{1}\right),\left(\hat{\beta}_{2}-\beta_{2}\right)\right) \rightarrow N_{3}\left(0, I^{-1}\left(\alpha, \beta_{1}, \beta_{2}\right)\right),
$$

where $I^{-1}\left(\alpha, \beta_{1}, \beta_{2}\right)$ is the variance-covariance matrix of the vector of unknown parameters. In practice, we usually estimate $I^{-1}\left(\alpha, \beta_{1}, \beta_{2}\right)$ by $I_{0}^{-1}\left(\hat{\alpha}, \hat{\beta}_{1}, \hat{\beta}_{2}\right)$

$$
I_{0}=-\left[\frac{\partial^{2} L}{\partial \theta_{s} \partial \theta_{l}}\right] \cdot s, l=1,2,3,
$$

where $\theta=\left(\alpha, \beta_{1}, \beta_{2}\right)$.

Therefore, the approximate $100(1-\gamma) \%$ two sided confidence intervals for $\alpha, \beta_{1}$ and $\beta_{1}$ are, respectively, given by

$$
\begin{aligned}
& \hat{\alpha} \mp Z_{\gamma / 2} \sqrt{I_{11}^{-1}\left(\hat{\alpha}, \hat{\beta}_{1}, \hat{\beta}_{2}\right)}, \\
& \hat{\beta}_{1} \mp Z_{\gamma / 2} \sqrt{I_{22}^{-1}\left(\hat{\alpha}, \hat{\beta}_{1}, \hat{\beta}_{2}\right)}, \\
& \hat{\beta}_{2} \mp Z_{\gamma / 2} \sqrt{I_{33}^{-1}\left(\hat{\alpha}, \hat{\beta}_{1}, \hat{\beta}_{2}\right)} .
\end{aligned}
$$

Here, $Z_{\gamma / 2}$ is the upper $(\gamma / 2)$ th percentile of standard normal distribution.

\subsection{Bootstrap Confidence Intervals}

In this subsection, we propose to use two confidence intervals based on the parametric bootstrap methods: (i) percentile bootstrap method (Boot-p) based on the idea of Efron [19]. (ii) bootstrap-t method (Boot-t) based on the idea of Hall [20]. The confidence intervals of $\theta=\left(\alpha, \beta_{1}, \beta_{2}\right)$ using both methods are illustrated briefly in the following steps:

Step 1: From the original data $\underline{x} \equiv\left(\left(x_{1: m: n}, \quad \delta_{1}, R_{1}\right)\right.$, $\left.\left(x_{2: m: n}, \delta_{2}, R_{2}\right), \cdots,\left(x_{m: m: n}, \quad \delta_{m}, R_{m}\right)\right)$ compute the MLE's of the parameters: say $\hat{\alpha}, \hat{\beta}_{1}$ and $\hat{\beta}_{2}$, equations $(12,13,16)$.

Step 2: Use $\hat{\alpha}, \hat{\beta}_{1}$ and $\hat{\beta}_{2}$ in Step 1 to generate a bootstrap sample $\underline{x}^{*}$ with the same values of $R_{i}, k, m$ and $n$ , $(i=1,2, . ., m)$. We used the algorithm proposed by Balakrishnan and Sandhu [21], with the fact that, the progressive first-failure censored sample with distribution function $F(x)$, can be viewed as a progressive Type II censored sample from a population with distribution function $1-(1-F(x))^{k}, m_{i} \sim \operatorname{Bin}\left(m, \frac{\beta_{i}}{\beta_{1}+\beta_{2}}\right), \quad i=1,2$.

Step 3: Repeat Step 2, $B$ times representing $\hat{\phi}_{l}^{*(1)}, \hat{\phi}_{l}^{*(2)}, \cdots, \hat{\phi}_{l}^{*(B)}, l=1,2,3$, where $\hat{\phi}_{1}^{*}=\hat{\alpha}^{*}, \hat{\phi}_{2}^{*}=\hat{\beta}_{1}^{*}$ and $\hat{\phi}_{4}^{*}=\hat{\beta}_{2}^{*}$.

Step 4: Arrange all $\hat{\phi}_{l}^{*(1)}, \hat{\phi}_{l}^{*(2)}, \cdots, \hat{\phi}_{l}^{*(B)}$ in an ascending order to obtain the bootstrap sample $\hat{\phi}_{l(1)}^{*}, \hat{\phi}_{l(2)}^{*}, \cdots, \hat{\phi}_{l(B)}^{*}$.

I- Percentile bootstrap method (Boot-p)

Let $G(x)=P\left(\hat{\phi}_{l}^{*} \leq x\right)$ be the cdf of $\hat{\phi}_{l}^{*}$. Define 
$\phi_{l, \text { boot-p }}=G^{-1}(x)$ for given $x$. The approximate bootstrap $100(1-\gamma) \%$ confidence interval of $\phi_{l}$ are given by

$$
\left[\phi_{l, \text { Boot-p }}\left(\frac{\gamma}{2}\right), \phi_{l, \text { Boot-p }}\left(1-\frac{\gamma}{2}\right)\right] \text {. }
$$

\section{II- Bootstrap-t method (Boot- $t$ )}

Compute the following statistic:

$$
T_{l}^{*}=\frac{\sqrt{m}\left(\hat{\phi}_{l}^{*}-\hat{\phi}_{l}\right)}{\sqrt{\operatorname{Var}\left(\hat{\phi}_{l}^{*}\right)}}, \quad l=1,2,3,
$$

where $\operatorname{Var}\left(\hat{\phi}_{l}^{*}\right)$ are obtained using the Fisher Information matrix. Using $T_{l}^{*}$ values, determine the upper and lower bounds of the $100(1-\gamma) \%$ confidence interval of $\phi_{l}$ as follows: let $H(x)=P\left(T_{l}^{*} \leq x\right), l=1,2,3$ be the cdf of $T_{l}^{*}$. For a given $x$, define

$$
\hat{\phi}_{l, \text { Boot-t }}(x)=\hat{\phi}_{l}+m^{-1 / 2} \sqrt{\operatorname{Var}\left(\hat{\phi}_{l}\right)} H^{-1}(x) \text {, for } l=1,2,3 \text {. }
$$

Here also, $\operatorname{Var}\left(\hat{\phi}_{l}\right)$ can be computed as same as computing the $\operatorname{Var}\left(\hat{\phi}_{l}^{*}\right)$. The approximate $100(1-\gamma) \%$ confidence interval of $\phi_{l}$ is given by

$$
\left(\hat{\phi}_{l, \text { Boot }-t}\left(\frac{\gamma}{2}\right), \hat{\phi}_{l, \text { Boot-t }}\left(1-\frac{\gamma}{2}\right)\right) \text {, for } l=1,2,3
$$

Hall (1988) showed that the Boot-t confidence interval is better than the Boot-p confidence interval from an asymptotic point of view.

\section{Data Analysis}

We consider in this section a real-life data set which was originally reported by Hoel [17] and latter analyzed by several authors, see for example Pareek et al. [22], Sarhan et al. [4] and Cramer and Schmiedt [3]. It was obtained from a laboratory experiment in which male mice received a radiation dose of 300 roentgens. The cause of death for each mouse was determined by autopsy. Restricting the analysis to two causes of death, for the purpose of analysis, we consider thymic lymphoma as cause 1 and combine the other causes of death as cause 2 . There were $m_{1}=29$ deaths due to cause 1 and $m_{1}=39$ deaths due to cause 2 .

The mean, standard deviation and the coefficient of skewness for the two causes of death are calculated (344.034, 170.568, 1.106) and (412.923, 203.518, - 0.227 ), respectively. The measure of skewness indicates that the data are positively and negatively skewed for cause 1 and cause 2 , respectively. For computational ease, we have divided each data point by 1000 .
To check the validity of the model, we compute the the Kolmogorov Smirnov (K-S) statistic whether the Burr XII model is suitable for this data. The maximum likelihood estimates of $\alpha$ and $\beta$ based on the two causes of death are $(2.339,9.819)$ and $(2.226,6.214)$, respectively. In deaths

due to cause 1 the K-S distance and the associated p-value are 0.182 and 0.290 , respectively, and for the deaths due to cause 2 the corresponding values are 0.109 and 0.740 . Based on the p-values, the Burr XII model is found to fit the data well. We have plotted the empirical survival functions, and the fitted survival functions in Fig. 1 and Fig. 2 for both data sets. Observe that they fit the data very well.

Now, there were $n=68$ observations in the data. The data are randomly grouped into 17 groups with $(k=4)$ items within each group. We suppose that the pre-determined progressively first-failure censored scheme is given by ( $R_{1}=R_{2}=2, \quad R_{3}=R_{4}=\cdots=R_{13}=0$ ), then a progressively first-failure censored competing risks data of size 13 out of 17 groups of death time is obtained as $(0.040,2), \quad(0.158,1), \quad(0.195,1), \quad(0.222,2), \quad(0.244,1)$, $(0.282,2), \quad(0.431,2), \quad(0.434,1), \quad(0.482,2), \quad(0.529,1)$, $(0.564,2), \quad(0.620,2), \quad(0.651,2)$.

There were $m_{1}=5$ deaths due to cause 1 and $m_{1}=8$ deaths due to cause 2 . We would like to compute the MLE's of the unknown parameters. Before going to compute the MLE's, we plot the profile log-likelihood function (14) in Fig. 3. From the Fig. 3 it is clear that the profile log-likelihood function is unimodal and the MLE of $\alpha$ is close to 2.1. We start the iteration to solve the Eq. (15) with $\alpha=2.1$, and obtain the $\hat{\alpha}=2.2347, \hat{\beta}_{1}=0.7122$ and $\hat{\beta}_{2}=1.1396$, and the $95 \%$ confidence intervals for $\alpha, \beta_{1}$ and $\beta_{2}$ are $(1.3263,3.143)$, $(-0.0419,1.4664),(0.0995,2.1797)$, the Boot-p confidence intervals are $\quad(1.6287,3.1321), \quad(0.2287,1.6598)$, $(0.5133,1.9044)$ and the corresponding Boot-t confidence intervals are $(1.3338,2.9222), \quad(-0.4838,1.1396)$, $(-0.2395,1.5838)$, respectively. Using the two confidence intervals (Boot-p) and (Boot-t), we present the mean of 1000 bootstrap samples of $\left(\alpha, \beta_{1}, \beta_{2}\right)$ by $\left(\hat{\alpha}, \hat{\beta}_{1}, \hat{\beta}_{2}\right)$.

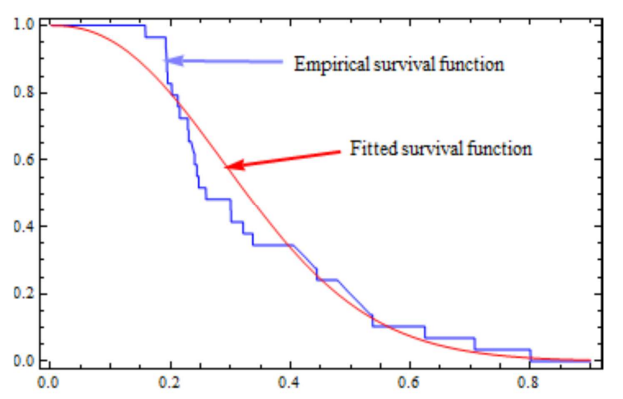

Fig. 1. The empirical and fitted survival functions for deaths due to cause 1 . 


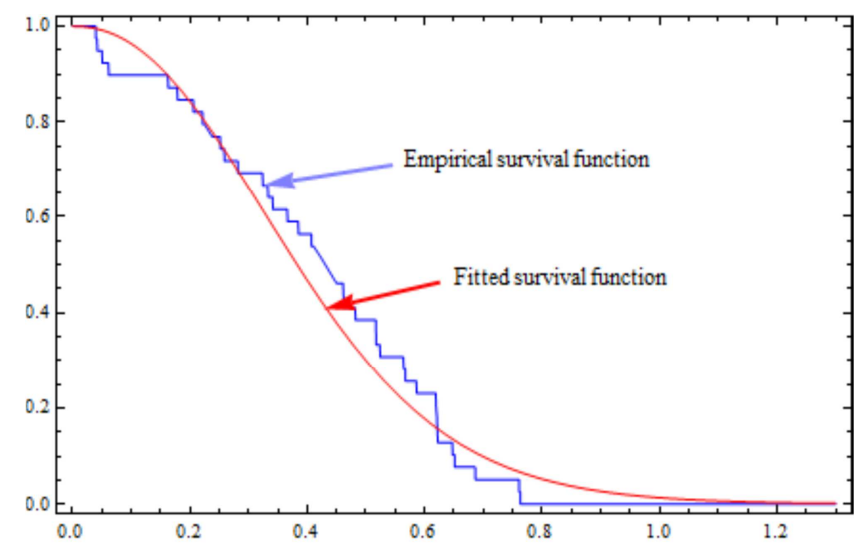

Fig. 2. The empirical and fitted survival functions for deaths due to cause 2 .

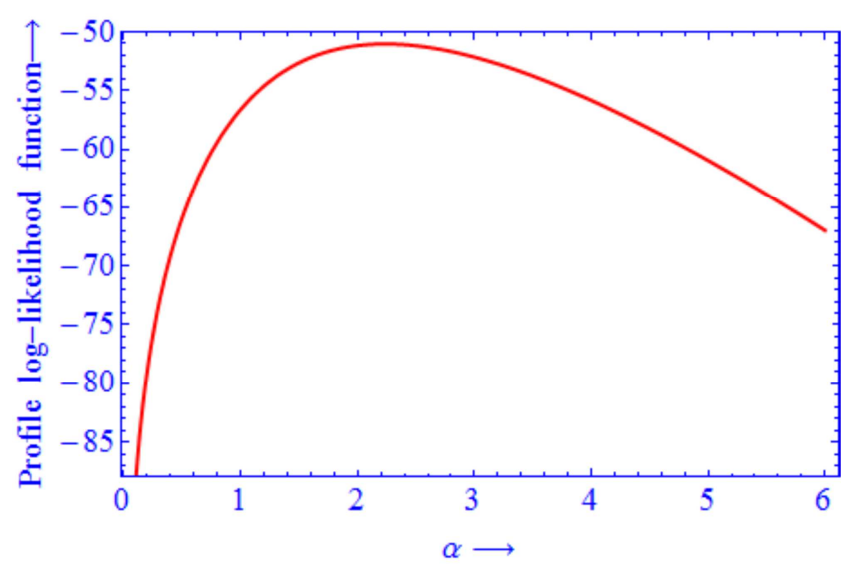

Fig. 3. The profile log-likelihood function (14).

\section{Monte Carlo Simulations}

In this section we primarily perform some simulation experiments to observe the behavior of the different methods. Monte Carlo simulations were performed utilizing 1000 progressively first-failure-censored samples for each simulations. The samples were generated by using the algorithm described in Balakrishnan and Sandh [20] using $\left(\alpha, \beta_{1}, \beta_{2}\right)=(1.5,0.3,0.7),(1.5,0.4,0.5)$ with different choices of $n, m$ and $k$. We take into consideration that the progressively first-failure-censored order statistics $x_{1: m: n: k}<x_{2: m: n: k}<\cdots<x_{m: m: n: k} \quad$ is a progressively Type II censored sample from a population with distribution function $1-(1-F(x))^{k}$. For each data point, we assigned the cause of failure as 1 or 2 with probability $\left(\beta_{1} /\left(\beta_{1}+\beta_{2}\right)\right)$ and $\left(\beta_{2} /\left(\beta_{1}+\beta_{2}\right)\right)$, respectively. We consider the following different sampling schemes:

Scheme I : $R_{1}=n-m, R_{i}=0$ for $i \neq 1$.

Scheme II : $R_{\frac{m}{2}}=n-m, R_{i}=0$ for $i \neq \frac{m}{2}$.

Scheme III : $R_{m}=n-m, R_{i}=0$ for $i \neq m$.

The MLE $\hat{\alpha}$ of parameter $\alpha$ is then computed from the solution of Equation (15) using the Newton-Raphson iteration. Once we estimate $\alpha$, we derived $\hat{\beta}_{1}$ and $\hat{\beta}_{2}$ using (12) and (13), respectively. We compute the average estimates, mean squared errors (MSEs) of the MLE's. The estimated coverage probability, and the average lengths are computed for different methods of estimation. For both Boot-p and Boot-t, we considered 1000 replications. Tables 1-4 summarize the obtained results.

Table 1. The average estimates of $\alpha, \beta_{1}$ and $\beta_{2}$ and their mean squared errors (within brackets) of MLE's, for different censoring schemes ( $\alpha=1.5$, $\beta_{1}=0.3$ and $\left.\beta_{2}=0.7\right)$.

\begin{tabular}{|c|c|c|c|c|c|c|}
\hline k & $\mathbf{n}$ & m & Scheme & $\hat{\alpha}$ & $\hat{\beta}_{1}$ & $\hat{\beta}_{2}$ \\
\hline \multirow[t]{6}{*}{1} & 30 & 20 & I & $1.564(0.0981)$ & $0.3296(0.0155)$ & $0.8024(0.0433)$ \\
\hline & & & II & $1.6261(0.1150)$ & $0.2864(0.0156)$ & $0.6868(0.0533)$ \\
\hline & & & III & $1.6322(0.1184)$ & $0.2768(0.0196)$ & $0.7548(0.0649)$ \\
\hline & 40 & 30 & I & $1.5161(0.0485)$ & $0.3156(0.0134)$ & $0.7694(0.0234)$ \\
\hline & & & II & $1.5686(0.0615)$ & $0.2996(0.0158)$ & $0.7007(0.0334)$ \\
\hline & & & III & $1.6164(0.0854)$ & $0.3293(0.0189)$ & $0.7531(0.0418)$ \\
\hline \multirow[t]{6}{*}{5} & 30 & 20 & I & $1.6178(0.1081)$ & $0.3357(0.0325)$ & $0.7957(0.1045)$ \\
\hline & & & II & $1.5612(0.1093)$ & $0.3827(0.0478)$ & $0.8106(0.1319)$ \\
\hline & & & III & $1.6069(0.1098)$ & $0.3733(0.0432)$ & $0.9176(0.1702)$ \\
\hline & 40 & 30 & I & $1.5483(0.0574)$ & $0.3411(0.0176)$ & $0.7272(0.0349)$ \\
\hline & & & II & $1.5292(0.0678)$ & $0.3142(0.0179)$ & $0.7111(0.0418)$ \\
\hline & & & III & $1.5495(0.0758)$ & $0.3298(0.0188)$ & $0.7943(0.0470)$ \\
\hline
\end{tabular}


Table 2. The average estimates of $\alpha, \beta_{1}$ and $\beta_{2}$ and their mean squared errors (within brackets) of MLE's for different censoring schemes ( $\alpha=1.5$, $\beta_{1}=0.4$ and $\beta_{2}=0.5$ ).

\begin{tabular}{|c|c|c|c|c|c|c|}
\hline $\mathbf{k}$ & $\mathbf{n}$ & $\mathbf{m}$ & Scheme & $\hat{\alpha}$ & $\hat{\beta}_{1}$ & $\hat{\beta}_{2}$ \\
\hline \multirow[t]{6}{*}{1} & 30 & 20 & I & $1.5333(0.0559)$ & $0.4148(0.0164)$ & $0.5717(0.0245)$ \\
\hline & & & II & $1.5579(0.0655)$ & $0.4357(0.0241)$ & $0.5184(0.0292)$ \\
\hline & & & III & $1.5958(0.0898)$ & $0.4082(0.0321)$ & $0.5148(0.0286)$ \\
\hline & 40 & 30 & I & $1.5265(0.0403)$ & $0.3729(0.0088)$ & $0.5148(0.0224)$ \\
\hline & & & II & $1.6347(0.0599)$ & $0.4022(0.0161)$ & $0.4833(0.0263)$ \\
\hline & & & III & $1.5859(0.0665)$ & $0.4301(0.0214)$ & $0.5213(0.0282)$ \\
\hline \multirow[t]{6}{*}{5} & 30 & 20 & I & $1.5952(0.0753)$ & $0.5146(0.0208)$ & $0.5949(0.0535)$ \\
\hline & & & II & $1.5653(0.0778)$ & $0.4516(0.0297)$ & $0.5883(0.0644)$ \\
\hline & & & III & $1.4787(0.0836)$ & $0.4052(0.0350)$ & $0.5241(0.0673)$ \\
\hline & 40 & 30 & I & $1.5643(0.0494)$ & $0.4288(0.0160)$ & $0.5490(0.0400)$ \\
\hline & & & II & $1.5916(0.0488)$ & $0.4691(0.0298)$ & $0.6073(0.0520)$ \\
\hline & & & III & $1.6113(0.0715)$ & $0.4747(0.0325)$ & $0.5713(0.0567)$ \\
\hline
\end{tabular}

Table 3. The average $95 \%$ confidence lengths and the corresponding coverage percentages (within brackets) of Boot-p and Boot-t for different censoring schemes $\left(\alpha=1.5, \beta_{1}=0.3\right.$ and $\left.\beta_{2}=0.7\right)$.

\begin{tabular}{|c|c|c|c|c|c|c|c|}
\hline k & $\mathbf{n}$ & $\mathbf{m}$ & Scheme & Param. & MLE & Boot-p & Boot-t \\
\hline \multirow[t]{18}{*}{1} & 30 & 20 & I & $\alpha$ & $1.4588(0.964)$ & $1.4588(0.928)$ & $1.2507(0.945)$ \\
\hline & & & & $\beta_{1}$ & $0.4999(0.912)$ & $0.5810(0.912)$ & $0.5812(0.927)$ \\
\hline & & & & $\beta_{2}$ & $0.8354(0.934)$ & $0.8843(0.921)$ & $0.9085(0.923)$ \\
\hline & & & II & $\alpha$ & $1.4457(0.888)$ & $1.4457(0.896)$ & $1.2351(0.888)$ \\
\hline & & & & $\beta_{1}$ & $0.4758(0.864)$ & $0.5510(0.921)$ & $0.5510(0.911)$ \\
\hline & & & & $\beta_{2}$ & $0.7457(0.927)$ & $0.8240(0.932)$ & $0.7991(0.912)$ \\
\hline & & & III & $\alpha$ & $1.4263(0.912)$ & $1.4263(0.925)$ & $1.2102(0.905)$ \\
\hline & & & & $\beta_{1}$ & $0.5037(0.911)$ & $0.5821(0.903)$ & $0.5821(0.924)$ \\
\hline & & & & $\beta_{2}$ & $0.8011(0.922)$ & $0.9029(0.922)$ & $0.8634(0.918)$ \\
\hline & 40 & 30 & I & $\alpha$ & $1.1166(0.965)$ & $1.1166(0.974)$ & $0.9669(0.972)$ \\
\hline & & & & $\beta_{1}$ & $0.3926(0.962)$ & $0.4344(0.977)$ & $0.4344(0.969)$ \\
\hline & & & & $\beta_{2}$ & $0.6594(0.963)$ & $0.7080(0.968)$ & $0.7337(0.976)$ \\
\hline & & & II & $\alpha$ & $1.0653(0.952)$ & $1.0653(0.935)$ & $0.9448(0.955)$ \\
\hline & & & & $\beta_{1}$ & $0.4237(0.960)$ & $0.4384(0.961)$ & $0.4384(0.968)$ \\
\hline & & & & $\beta_{2}$ & $0.6399(0.975)$ & $0.7087(0.968)$ & $0.6703(0.794)$ \\
\hline & & & III & $\alpha$ & $1.0682(0.925)$ & $1.0682(0.950)$ & $0.9450(0.945)$ \\
\hline & & & & $\beta_{1}$ & $0.4168(0.954)$ & $0.4377(0.944)$ & $0.4377(0.948)$ \\
\hline & & & & $\beta_{2}$ & $0.6139(0.923)$ & $0.7168(0.932)$ & $0.6447(0.942)$ \\
\hline \multirow[t]{18}{*}{5} & 30 & 20 & I & $\alpha$ & $0.9763(0.922)$ & $0.9763(0.925)$ & $0.8815(0.935)$ \\
\hline & & & & $\beta_{1}$ & $0.5732(0.875)$ & $0.7319(0.936)$ & $0.7319(0.919)$ \\
\hline & & & & $\beta_{2}$ & $1.0132(0.925)$ & $1.1340(0.925)$ & $0.9459(0.933)$ \\
\hline & & & II & $\alpha$ & $0.9414(0.925)$ & $0.9414(0.900)$ & $0.8878(0.912)$ \\
\hline & & & & $\beta_{1}$ & $0.6696(0.915)$ & $0.8292(0.923)$ & $0.8292(0.931)$ \\
\hline & & & & $\beta_{2}$ & $1.3147(0.925)$ & $1.1868(0.922)$ & $1.2261(0.914)$ \\
\hline & & & III & $\alpha$ & $1.0996(0.901)$ & $1.0996(0.922)$ & $1.0624(0.918)$ \\
\hline & & & & $\beta_{1}$ & $0.8561(0.925)$ & $0.8888(0.925)$ & $0.8888(0.922)$ \\
\hline & & & & $\beta_{2}$ & $1.3223(0.911)$ & $1.2626(0.915)$ & $1.4501(0.914)$ \\
\hline & 40 & 30 & I & $\alpha$ & $0.8228(0.968)$ & $0.8228(0.968)$ & $0.7689(0.972)$ \\
\hline & & & & $\beta_{1}$ & $0.4541(0.964)$ & $0.5523(0.963)$ & $0.5523(0.967)$ \\
\hline & & & & $\beta_{2}$ & $0.7493(0.962)$ & $0.9130(0.965)$ & $0.7152(0.971)$ \\
\hline & & & II & $\alpha$ & $0.8159(0.954)$ & $0.8159(0.956)$ & $0.7519(0.958)$ \\
\hline & & & & $\beta_{1}$ & $0.4663(0.942)$ & $0.5855(0.947)$ & $0.5855(0.955)$ \\
\hline & & & & $\beta_{2}$ & $0.8456(0.955)$ & $0.9622(0.962)$ & $0.8253(0.949)$ \\
\hline & & & III & $\alpha$ & $0.9415(0.939)$ & $0.9415(0.942)$ & $0.8873(0.940)$ \\
\hline & & & & $\beta_{1}$ & $0.5782(0.941)$ & $0.6873(0.951)$ & $0.6873(0.943)$ \\
\hline & & & & $\beta_{2}$ & $1.0807(0.933)$ & $1.1088(0.947)$ & $1.0262(0.951)$ \\
\hline
\end{tabular}


Table 4. The average $95 \%$ confidence lengths and the corresponding coverage percentages (within brackets) of MLE's, Boot-p and Boot-t for different censoring schemes $\left(\alpha=1.5, \quad \beta_{1}=0.4\right.$ and $\left.\beta_{2}=0.5\right)$.

\begin{tabular}{|c|c|c|c|c|c|c|c|}
\hline $\mathbf{k}$ & $\mathbf{n}$ & $\mathbf{m}$ & Scheme & Param. & MLE & Boot-p & Boot-t \\
\hline \multirow[t]{18}{*}{1} & \multirow[t]{9}{*}{30} & \multirow[t]{9}{*}{20} & \multirow[t]{3}{*}{ I } & $\alpha$ & $1.5567(0.932)$ & $1.5567(0.931)$ & $1.3397(0.944)$ \\
\hline & & & & $\beta_{1}$ & $0.5637(0.920)$ & $0.5946(0.908)$ & $0.5946(0.926)$ \\
\hline & & & & $\beta_{2}$ & $0.5876(0.917)$ & $0.6729(0.925)$ & $0.6351(0.932)$ \\
\hline & & & \multirow[t]{3}{*}{ II } & $\alpha$ & $1.376(0.922)$ & $1.376(0.933)$ & $1.1531(0.940)$ \\
\hline & & & & $\beta_{1}$ & $0.5528(0.933)$ & $0.6282(0.935)$ & $0.6282(0.941)$ \\
\hline & & & & $\beta_{2}$ & $0.6335(0.930)$ & $0.7282(0.941)$ & $0.7348(0.938)$ \\
\hline & & & \multirow[t]{3}{*}{ III } & $\alpha$ & $1.5142(0.935)$ & $1.5142(0.914)$ & $1.2889(0.902)$ \\
\hline & & & & $\beta_{1}$ & $0.5492(0.921)$ & $0.6483(0.943)$ & $0.6483(0.928)$ \\
\hline & & & & $\beta_{2}$ & $0.6132(0.920)$ & $0.7154(0.932)$ & $0.6910(0.937)$ \\
\hline & \multirow[t]{9}{*}{40} & \multirow[t]{9}{*}{30} & \multirow[t]{3}{*}{ I } & $\alpha$ & $1.1650(0.965)$ & $1.1650(0.966)$ & $1.0368(0.977)$ \\
\hline & & & & $\beta_{1}$ & $0.4720(0.956)$ & $0.4999(0.976)$ & $0.4999(0.964)$ \\
\hline & & & & $\beta_{2}$ & $0.5205(0.954)$ & $0.5722(0.969)$ & $0.5616(0.975)$ \\
\hline & & & \multirow[t]{3}{*}{ II } & $\alpha$ & $1.1449(0.945)$ & $1.1449(0.947)$ & $1.0105(0.969)$ \\
\hline & & & & $\beta_{1}$ & $0.4529(0.962)$ & $0.4889(0.976)$ & $0.4889(0.974)$ \\
\hline & & & & $\beta_{2}$ & $0.5062(0.965)$ & $0.5453(0.977)$ & $0.5503(0.972)$ \\
\hline & & & \multirow[t]{3}{*}{ III } & $\alpha$ & $1.1082(0.925)$ & $1.1082(0.933)$ & $0.9798(0.934)$ \\
\hline & & & & $\beta_{1}$ & $0.4482(0.945)$ & $0.4936(0.940)$ & $0.4936(0.953)$ \\
\hline & & & & $\beta_{2}$ & $0.5062(0.937)$ & $0.5592(0.939)$ & $0.5625(0.947)$ \\
\hline \multirow[t]{18}{*}{5} & \multirow[t]{9}{*}{30} & \multirow[t]{9}{*}{20} & \multirow[t]{3}{*}{ I } & $\alpha$ & $0.9790(0.925)$ & $0.9790(0.941)$ & $0.8808(0.932)$ \\
\hline & & & & $\beta_{1}$ & $0.6335(0.933)$ & $0.7731(0.943)$ & $0.7731(0.939)$ \\
\hline & & & & $\beta_{2}$ & $0.6947(0.925)$ & $0.8827(0.937)$ & $0.689(0.957)$ \\
\hline & & & \multirow[t]{3}{*}{ II } & $\alpha$ & $1.0145(0.924)$ & $1.0145(0.933)$ & $0.9164(0.947)$ \\
\hline & & & & $\beta_{1}$ & $0.9672(0.936)$ & $0.9761(0.942)$ & $0.9761(0.944)$ \\
\hline & & & & $\beta_{2}$ & $1.1838(0.925)$ & $1.0647(0.936)$ & $1.1856(0.953)$ \\
\hline & & & \multirow[t]{3}{*}{ III } & $\alpha$ & $1.1527(0.924)$ & $1.1527(0.935)$ & $1.0459(0.932)$ \\
\hline & & & & $\beta_{1}$ & $0.9119(0.930)$ & $1.1457(0.933)$ & $1.1457(0.941)$ \\
\hline & & & & $\beta_{2}$ & $1.1208(0.941)$ & $1.2670(0.932)$ & $1.1463(0.939)$ \\
\hline & \multirow[t]{9}{*}{40} & 30 & \multirow[t]{3}{*}{ I } & $\alpha$ & $0.8551(0.965)$ & $0.8551(0.968)$ & $0.7849(0.977)$ \\
\hline & & & & $\beta_{1}$ & $0.5075(0.955)$ & $0.6412(0.971)$ & $0.6412(0.959)$ \\
\hline & & & & $\beta_{2}$ & $0.5831(0.965)$ & $0.7109(0.969)$ & $0.5871(0.967)$ \\
\hline & & & \multirow[t]{3}{*}{ II } & $\alpha$ & $0.8425(0.955)$ & $0.8425(0.966)$ & $0.7667(0.955)$ \\
\hline & & & & $\beta_{1}$ & $0.5389(0.952)$ & $0.6470(0.953)$ & $0.6470(0.976)$ \\
\hline & & & & $\beta_{2}$ & $0.5835(0.961)$ & $0.7474(0.971)$ & $0.5529(0.963)$ \\
\hline & & & \multirow[t]{3}{*}{ III } & $\alpha$ & $0.9334(0.944)$ & $0.9334(0.957)$ & $0.8541(0.966)$ \\
\hline & & & & $\beta_{1}$ & $0.6753(0.936)$ & $0.8376(0.963)$ & $0.8376(0.945)$ \\
\hline & & & & $\beta_{2}$ & $0.8328(0.946)$ & $0.9586(0.949)$ & $0.8335(0.962)$ \\
\hline
\end{tabular}

\section{Conclusions}

In this paper, we have analyzed progressive first-failure-censoring in the presence of competing risks. In particular, we have assumed that the latent failure times under the competing risks follow independent Burr XII distributions with common the shape parameters. We compared different statistical inference procedures and the performance of the unknown parameters based on MLE, Boot-p and Boot- $t$ methods in this setting. We have then conducted a simulation study to assess the performance of all these procedures and a numerical example has been presented to illustrate all the methods of inference developed in this paper. This work can be extended in several directions. Bayesian inference is also possible through the inclusion of suitable prior distributions. Inferences can be extended to allow for more than two causes. Based on the results of the simulation study some of the points are clear from this experiment. Even for some small sample sizes, we observe the following:

The results obtained in this paper can be specialized to: (a) First-failure-censored order statistics by taking $R=(0, \cdots, 0)$.

(b) Progressively Type II censored statistics for $k=1$. (c) Usually Type II censored order statistics for $k=1$ and $R=(0, \ldots, n-m)$. (d) Complete sample for $k=1, n=m$ and $R=(0, \cdots, 0)$. 
From Tables $1-4$, as expected for all the methods, when $n, m$ increase then the average lengths and the MSEs decrease.

From Tables $3-4$, in most cases the estimated coverage probability is close to the nominal level of 0.95 based on different methods.

We also observe very stable coverage probabilities (quite close to the nominal level). On the other hand, the performances of the MLE, Boot-p and Boot-t methods are satisfactory for small sample sizes as their actual coverage probabilities are close to the specified nominal levels in most cases.

\section{References}

[1] M. L. Moeschberger, K. P. Tordoff, N. Kochar, A review of statistical analyses for competing risks, in: Epidemiology and Medical Statistics. Vol. 27 of Handbook of Statist, Elsevier/North-Holland, Amsterdam, (2008), 321-341.

[2] F. Pascual, Accelerated life test planning with independent lognormal competing risks. Journal of Statistical Planning and Inference. 140 (4), (2010), 1089-1100.

[3] E. Cramer, A. B. Schmiedt, Progressively Type-II censored competing risks data from Lomax distributions. Computational Statistics and Data Analysis. 55, (2011), 1285-1303.

[4] A. M. Sarhan, D. C. Hamilton, B. Smith, Statistical analysis of competing risks models. Reliability Engineering and System Safety. 95, (2010), 953-962.

[5] A. M. Sarhan, Analysis of incomplete, censored data in competing risks models with generalized exponential distributions. IEEE Transactions on Reliability. 56, (2007), 102-107.

[6] I. A. Alwasel, Statistical inference of a competing risks model with modified Weibull distributions. International Journal of Mathematical Analysis. 3, (2009), 905-918.

[7] D. Kundu, S. Basu, Analysis of incomplete data in presence of competing risks. Journal of Statistical Planning and Inference. 87, (2000), 221-239.

[8] D. Kundu, A. M. Sarhan, Analysis of incomplete data in the presence of competing risks among several groups. IEEE Transactions on Reliability. 55, (2006), 262-269.

[9] J.-W. Wu, W.-L. Hung, C.-H. Tsai, Estimation of the parameters of the Gompertz distribution under the first-failure-censored sampling plan. Statistics. 37 (6), (2003), 517-525.
[10] J.-W. Wu, H.-Y. Yu, Statistical inference about the shape parameter of the Burr type XII distribution under the failure-censored sampling plan. Applied Mathematics and computation. 163, (2005), 443-482.

[11] L. G. Johnson, Theory and Technique of Variation Research. Elsevier, Amsterdam, (1964).

[12] S.-J. Wu, C. Kuş, On estimation based on progressive first-failure-censored sampling. Computational Statistics and Data Analysis. 53 (10), (2009), 3659-3670.

[13] A. A. Soliman, A. H. Abd Ellah, N. A. Abou-Elheggag, G. A. Abd-Elmougod, A simulation-based approach to the study of coefficient of variation of Gompertz distribution under progressive first-failure censoring. Indian Journal of Pure and Applied Mathematics. 42(5), (2011), 335-356.

[14] A. A. Soliman, A. H. Abd Ellah, N. A. Abou-Elheggag, G. A. Abd-Elmougod, Estimation of the parameters of life for Gompertz distribution using progressive first-failure censored data. Computational Statistics and Data Analysis. 56, (2012), 2471-2485.

[15] A. A. Soliman, A. H. Abd Ellah, N. A. Abou-Elheggag, A. A. Modhesh, Estimation of the coefficient of variation for non-normal model using progressive first-failure-censoring data. Journal of Applied Statistics. 39(12), (2012), 2741-2758.

[16] A. A. Soliman, A. H. Abd Ellah, N. A. Abou-Elheggag, A. A. Modhesh, Estimation from Burr type XII distribution using progressive first-failure censored data. Journal of Statistical Computation and Simulation. 83(12), (2013), 2270-2290.

[17] M. V. Ahmadi, M. Doostparast, J. Ahmadi, Estimating the lifetime performance index with Weibull distribution based on progressive first-failure censoring scheme. Journal of Computational and Applied Mathematics. 239, (2013), 93-102.

[18] D. G. Hoel, A representation of mortality data by competing risks. Biometrics. 28, (1972), 475-488.

[19] B. Efron, The jackknife, the bootstrap and other resampling plans. In: CBMS-NSF Regional Conference Seriesin Applied Mathematics, vol 38, SIAM, Philadelphia, PA, (1982).

[20] P. Hall, Theoretical comparison of bootstrap confidence intervals. Annals of Statistics. 16, (1988), 927-953.

[21] N. Balakrishnan, R. A. Sandhu, A simple simulation algorithm for generating progressively Type-II censored samples. The American Statistician. 49, (1995), 229-230.

[22] B. Pareek, D. Kundu, S. Kumar, On progressively censored competing risks data for Weibull distributions. Computer Statistics and Data Analysis. 53, (2009), 4083-4094. 